\title{
A LORENTZ FORM ASSOCIATED TO CONTACT SUB-CONFORMAL AND CR MANIFOLDS
}

\author{
Elisha Falbel and José Miguel Veloso
}

\begin{abstract}
We construct a bilinear form associated to a sub-conformal structure on a manifold $M$. In the case the sub-conformal structure corresponds to a partially-integrable CR structure we obtain a conformal Lorentz structure which coincides with Fefferman's construction on a circle bundle over $M$. The main contribution is the use of invariant forms with values in a vector space instead of the full information contained in the Cartan connection in order to simplify the construction.
\end{abstract}

\section{Introduction}

Associated to an integrable CR manifold $M$, that is a contact manifold with a complex structure defined in the contact distribution satisfying an integrability condition (see section 2 for definitions), one can define a Lorentz conformal structure on a circle bundle over $M$. That structure was first constructed for real hypersurfaces in $\mathbf{C}^{n}$ in $[\mathrm{F}]$ and later for an abstract $\mathrm{CR}$ manifold in [BDS].

The construction in $[\mathrm{BDS}]$ (see also $[\mathrm{Ca}]$ for a generalization) uses a Cartan connection obtained on a principal bundle canonically associated to a CR manifold with structure group $H_{C R}=C U(n) \ltimes N$ which is the automorphism group of the Heisenberg group $N$ (see [C, CM, T2] and section 2.4). The construction of that connection is not easy and therefore it was natural to look for other constructions.

In $[\mathrm{Fa}, \mathrm{L}]$ more elementary constructions are given in the sense that one does not need to use a connection associated to the CR structure. In particular, [L] uses Webster's connection associated to a pseudo-Hermitian structure (see [D] for a recent survey).

A natural generalization of CR geometry on a contact manifold is subconformal geometry which is a conformal structure defined on the contact distribution. That structure contains a large class of non-integrable CR manifolds called partially-integrable (see section 2).

2010 Mathematics Subject Classification. primary 32V05, secondary 53C17, 53D10, 58A15. Key words and phrases. CR structures, sub-Riemannian manifolds, Fefferman conformal metric. Received July 30, 2013. 
The goal of this paper is to construct a canonical bilinear form associated to a sub-conformal structure without any integrability conditions. In the case of integrable CR structures the bilinear form coincides with the one defined in [BDS] (cf. [FV] to compare Chern's construction and the parallelism defined for a subconformal structure).

The main observation is that only part of the Cartan connection for CR manifolds is needed to construct the bilinear form giving rise to the Lorentz structure. All relevant information is contained in an invariant form with values in a vector space $v_{C R}$ which is a quotient of the Lie algebra $s u(n+1,1)$ associated to $\mathrm{CR}$ manifolds. Instead of constructing the Cartan connection, we construct an invariant form with values in $v_{C R}$ (see section 2 for definitions). That construction is carried through in general for sub-conformal structures and the exposition is self-contained without any use of the CR connection, except for comparison purposes. From the construction of that invariant form we are able to characterize a Lorentzian conformal structure on a circle bundle over a partially-integrable CR manifold. The main result is Theorem 4.1. We use moving frames following $[\mathrm{CM}]$ which is appropriate for explicit calculations.

The authors thank the University of Paris VI and the University Federal of Pará (UFPA) for generous support while preparing this work.

The authors were partially supported by the Programa Nacional de Cooperação Acadêmica da Coordenação de Aperfeiçoamento de Pessoal de Nível Superior-CAPES/Brasil and by the 'Réseau Franco-Brésilien en Mathématiques'.

\section{CR-manifolds, sub-conformal manifolds and fiber bundles}

In this section we define the geometric structures we will deal with, namely CR structures and sub-conformal structures. Let $D$ be a contact distribution on a manifold $M$.

DEFINITION 2.1.

1. $(M, D, J)$ is an almost $\mathrm{CR}$ structure if $J: D \rightarrow D$ satisfies $J^{2}=-I$.

2. $(M, D, g)$ is a sub-Riemannian structure if $g$ is a metric on $D$.

3. $(M, D, \tilde{g})$ is a sub-conformal structure if $\tilde{g}$ is a conformal class of subRiemannian metrics.

Let $\pi: T M \rightarrow T M / D$ be the quotient map.

Definition 2.2. The Levi form $\alpha: D \times D \rightarrow T M / D$ is the skew-symmetric form defined as $\alpha(X, Y)=-\pi([X, Y])$.

Fixing a base $v$ of $T M / D$ defines the Levi form $\alpha_{v}$ as a real valued form. Let $\theta_{v}$ be the contact form of this distribution such that $\theta_{v}\left(\pi^{-1} v\right)=1$, then the Levi form is given by

$$
d \theta_{v}(X, Y)=\alpha_{v}(X, Y)
$$


If we have a metric on $D$, define a skew-symmetric operator $H_{v}$ on the distribution by

$$
\alpha_{v}(X, Y)=g\left(H_{v} X, Y\right)
$$

As $\alpha_{v}$ is non-degenerate, we can always choose $v$ such that det $H_{v}=1$ and this determines a unique $v$ ignoring orientation effects. Observe that if we let $t g$ be a new metric and choose $v$ as above, then

$$
\alpha_{(1 / t) v}(X, Y)=\operatorname{tg}\left(H_{v} X, Y\right)
$$

so the definition of $H_{v}$ does not depend on a metric inside a conformal class of metrics. Fixing a metric on $D$, denote by $H$ this operator and obtain its normal forms in the following lemma.

LeMma 2.1. Let $V$ be a $2 n$ dimensional real vector space with a scalar product. If $H$ is a nondegenerated skew-symmetric operator, then there exists an orthonormal basis of $V$ such that the matrix of $H$ is

$$
\left.\Lambda=\left(\begin{array}{ccc}
-\lambda_{1} & 0 & 0 \\
0 & \ddots & 0 \\
0 & 0 & -\lambda_{n}
\end{array}\right)\left(\begin{array}{ccc}
\lambda_{1} & 0 & 0 \\
0 & \ddots & 0 \\
0 & 0 & \lambda_{n}
\end{array}\right)\right)
$$

with $\lambda_{i}>0, i=1, \ldots, n$.

Lemma 2.2. Suppose that $\Lambda$ is such that $\lambda_{d_{1}+\cdots+d_{k-1}+1}=\cdots=\lambda_{d_{1}+\cdots+d_{k}}=v_{k}$ for $1 \leq k \leq r$, with $d_{1}+\cdots+d_{r}=n$, and $v_{1}<v_{2}<\cdots<v_{r}$, where the $v_{k}$ are real numbers, and that $A \in S O(2 n)$ satisfies $A \Lambda A^{T}=\Lambda$. Then $A \in U\left(d_{1}\right) \times \cdots \times$ $U\left(d_{r}\right)$.

Proof. We write $A=\left(a_{i}^{j}\right)$, and $1 \leq \alpha, \beta \leq n$. We have

$$
\begin{gathered}
a_{\alpha}^{\beta+n} \lambda_{\beta}=-a_{\alpha+n}^{\beta} \lambda_{\alpha} \\
a_{\alpha}^{\beta} \lambda_{\beta}=a_{\alpha+n}^{\beta+n} \lambda_{\alpha} \\
a_{\alpha+n}^{\beta+n} \lambda_{\beta}=a_{\alpha}^{\beta} \lambda_{\alpha} \\
a_{\alpha+n}^{\beta} \lambda_{\beta}=-a_{\alpha}^{\beta+n} \lambda_{\alpha}
\end{gathered}
$$

then

$$
\begin{gathered}
\lambda_{\alpha} \lambda_{\beta} a_{\alpha}^{\beta+n}=-\lambda_{\alpha}^{2} a_{\alpha+n}^{\beta}=-\lambda_{\beta}^{2} a_{\alpha+n}^{\beta} \\
\lambda_{\alpha} \lambda_{\beta} a_{\alpha}^{\beta}=\lambda_{\alpha}^{2} a_{\alpha+n}^{\beta+n}=\lambda_{\beta}^{2} a_{\alpha+n}^{\beta+n}
\end{gathered}
$$

If $\lambda_{\alpha} \neq \lambda_{\beta}$, we deduce $a_{\alpha}^{\beta}=a_{\alpha+n}^{\beta+n}=a_{\alpha}^{\beta+n}=a_{\alpha}^{\beta+n}=0$. If $\lambda_{\alpha}=\lambda_{\beta}$ then $a_{\alpha}^{\beta}=a_{\alpha+n}^{\beta+n}$, $a_{\alpha}^{\beta+n}=-a_{\alpha+n}^{\beta}$. Therefore $A \in U\left(d_{1}\right) \times \cdots \times U\left(d_{r}\right)$. 
A special case is when $\Lambda=J$. This clearly relates to CR manifolds and to be more explicit we introduce the next definitions.

DEFINITION 2.3.

1. An almost CR structure is partially-integrable if the Levi form satisfies the condition $\alpha(X, Y)=\alpha(J X, J Y)$.

2. A partially-integrable CR structure is integrable if $J([J X, J Y]-[X, Y])=$ $[J X, Y]+[X, J Y]$.

Essentially, the Levi form of a partially-integrable CR structure is compatible with the complex structure. Observe that a conformal sub-Riemannian manifold $(M, D, \tilde{g})$ such that $H^{2}=-I d$ is a partially-integrable CR manifold. In fact,

$$
\alpha(H X, H Y)=g\left(H^{2} X, H Y\right)=-g(X, H Y)=-g(H Y, X)=-\alpha(Y, X)=\alpha(X, Y) .
$$

Remark. The partially-integrable CR manifolds obtained above are strictly pseudoconvex. If we had started with conformal classes of pseudo-metrics $\tilde{g}$, we would have arrived at pseudoconvex CR manifolds. To see that, recall that the Levi form is extended as a Hermitian form on $D^{1,0}$, the eigenvectors of $H$ acting on $D \otimes \mathbf{C}$ corresponding to the eigenvalue $i$. Using an orthonormal basis $X_{\alpha}$, $H X_{\alpha}$ for $g$ which puts $H$ in normal form we have

$$
\alpha\left(X_{\alpha}-i H X_{\alpha}, X_{\alpha}+i H X_{\alpha}\right)=-2 i \alpha\left(H X_{\alpha}, X_{\alpha}\right)=-2 i g\left(H^{2} X_{\alpha}, X_{\alpha}\right)=2 i g\left(X_{\alpha}, X_{\alpha}\right),
$$

we see that the signature of $g$ corresponds to the signature of the Levi form. We will restrict our work to sub-Riemannian metrics for simplicity although the same results hold for different signatures.

2.1. $S U(n+1,1)$

We will use Chern's conventions $[\mathrm{CM}]$ (see also $[\mathrm{BDS}]$ ) where

$$
S U(n+1,1)=\left\{g \in S L(n+2, \mathbf{C}) \mid \bar{g}^{T} Q g=Q\right\}
$$

and the Hermitian form $Q$ is given by

$$
Q=\left(\begin{array}{ccc}
0 & 0 & -i / 2 \\
0 & I & 0 \\
i / 2 & 0 & 0
\end{array}\right) .
$$

The group $S U(n+1,1)$ acts on $\mathbf{C}^{n+2}$ on the left preserving the cone

$$
\left\{z \in \mathbf{C}^{n+2} \mid \bar{z}^{T} Q z=0\right\} .
$$

The projectivization of this cone is $S^{2 n+1} \subset \mathbf{C} P^{n+1}$. $\quad S U(n+1,1)$ acting on the sphere $S^{2 n+1}$ has a finite center $K$ which is a cyclic group of order $n+2$ acting trivially. We define $P U(n+1,1)=S U(n+1,1) / K$. 
A LORENTZ FORM ASSOCIATED TO CONTACT SUB-CONFORMAL AND CR MANIFOLDS 409

The elements of the Lie algebra $s u(n+1,1)$ are represented by the matrices

$$
\left(\begin{array}{ccc}
u & x & z \\
-2 i \bar{y}^{T} & A & 2 i \bar{x}^{T} \\
w & y & -\bar{u}
\end{array}\right)
$$

where $A$ is skew-Hermitian, $z, w \in \mathbf{R}, x, y \in \mathbf{C}^{n}, u \in \mathbf{C}$ and $u-\bar{u}+\operatorname{Tr} A=0$. Observe that the Lie algebra $g_{C R}=s u(n+1,1)$ is graded:

$$
g_{C R}=g^{-2} \oplus g^{-1} \oplus g^{0} \oplus g^{1} \oplus g^{2}
$$

where

$$
\begin{gathered}
g^{-2}=\left\{\left(\begin{array}{lll}
0 & 0 & z \\
0 & 0 & 0 \\
0 & 0 & 0
\end{array}\right)\right\} g^{-1}=\left\{\left(\begin{array}{ccc}
0 & x & 0 \\
0 & 0 & 2 i \bar{x}^{T} \\
0 & 0 & 0
\end{array}\right)\right\} \\
g^{0}=\left\{\left(\begin{array}{ccc}
u & 0 & 0 \\
0 & A & 0 \\
0 & 0 & -\bar{u}
\end{array}\right)\right\} g^{1}=\left\{\left(\begin{array}{ccc}
0 & 0 & 0 \\
-2 i \bar{y}^{T} & 0 & 0 \\
0 & y & 0
\end{array}\right)\right\} g^{2}=\left\{\left(\begin{array}{lll}
0 & 0 & 0 \\
0 & 0 & 0 \\
w & 0 & 0
\end{array}\right)\right\}
\end{gathered}
$$

Observe that

$$
g^{0}=\mathbf{R} \oplus u(n)=\mathbf{R} \oplus u(1) \oplus s u(n)
$$

where

$$
u(1)=\left\{\left(\begin{array}{ccc}
-i q / 2 & 0 & 0 \\
0 & i q I / n & 0 \\
0 & 0 & -i q / 2
\end{array}\right)\right\}
$$

with $q \in \mathbf{R}$ and

$$
\operatorname{csu}(n)=\mathbf{R} \oplus \operatorname{su}(n)=\left\{\left(\begin{array}{ccc}
r & 0 & 0 \\
0 & A & 0 \\
0 & 0 & -r
\end{array}\right)\right\}
$$

with $\operatorname{Tr} A=0$ and $r \in \mathbf{R}$.

Definition 2.4. We define the subalgebras

$$
\begin{array}{cc}
h_{C R}=g^{0} \oplus g^{1} \oplus g^{2} & h_{0}=u(n) \oplus g^{1} \oplus g^{2} \\
h_{1}=\operatorname{csu}(n) \oplus g^{1} \oplus g^{2} & h_{2}=s u(n) \oplus g^{1} \oplus g^{2}
\end{array}
$$

and the vector space

$$
v_{C R}=g_{C R} / h_{1} \simeq g^{-2} \oplus g^{-1} \oplus i \mathbf{R} .
$$

The isotropy of the action of $S U(n+1,1) / K$ on $S^{2 n+1}$ at the point $[0, \ldots, 1]^{T}$ is the group $H_{C R}=C U(n) \ltimes N$ (whose Lie algebra is $h_{C R}$ ), where $N$ is the Heisenberg group, represented (up to $K$ ) by matrices of the form 


$$
\left(\begin{array}{ccc}
a & 0 & 0 \\
-2 i a A \bar{b}^{T} & A & 0 \\
a\left(s-i b \bar{b}^{T}\right) & b & \bar{a}^{-1}
\end{array}\right)
$$

where $s \in \mathbf{R}, A \in U(n), b \in \mathbf{C}^{n}$ and $a \bar{a}^{-1} \operatorname{det} A=1$.

We follow the notations in [BDS] to define the following subgroups of $H_{C R}$; $H_{1}=C S U(n) \ltimes N$ (whose Lie algebra is $\left.h_{1}\right), H_{0}=U(n) \ltimes N$ (whose Lie algebra is $\left.h_{0}\right)$ and $H_{2}=S U(n) \ltimes N$ (whose Lie algebra is $h_{2}$ ). The inclusions between these groups can be visualized in the following diagrams

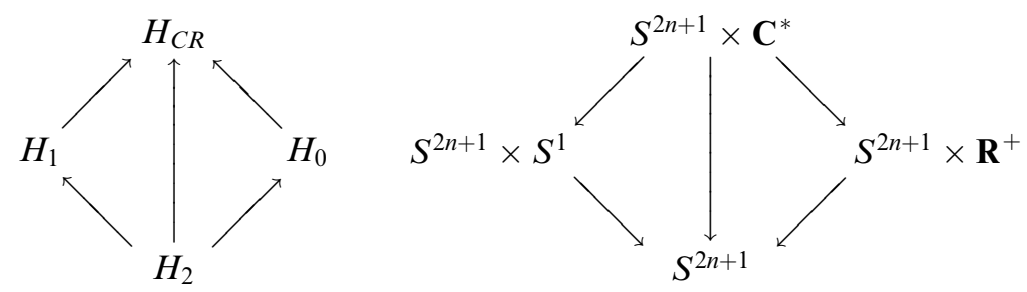

A geometrical interpretation of $S U(n+1,1) / H_{2}$ is obtained considering the canonical bundle of the sphere, that is the restriction of $\Lambda^{n+1} \mathbf{C}^{n+1}$ to the sphere, and deleting from it the zero section. This is a trivial $\mathbf{C}^{*}$ bundle over the sphere (it has a non-zero section given by the restriction of $d z^{1} \wedge \cdots \wedge d z^{n+1}$ to the sphere). We obtain $S U(n+1,1) / H_{2} \simeq S^{2 n+1} \times \mathbf{C}^{*}$ and also $S U(n+1,1) / H_{1} \simeq S^{2 n+1} \times S^{1}$, $S U(n+1,1) / H_{0} \simeq S^{2 n+1} \times \mathbf{R}^{+}$.

We will usually use the same notation for a lift of a subgroup of $P U(n+1,1)$ to $S U(n+1,1)$. In particular we will use the same notation for $H_{C R}$ as a subgroup of the matrix group $S U(n+1,1)$. The adjoint action of $H_{C R}$ on $g_{C R}$ is given by

$$
\begin{aligned}
& \left(\begin{array}{ccc}
a^{-1} & 0 & 0 \\
2 i \bar{b}^{T} & A^{-1} & 0 \\
-\bar{a}\left(s+i b \bar{b}^{T}\right) & -\bar{a} b A^{-1} & \bar{a}
\end{array}\right)\left(\begin{array}{ccc}
u & x & z \\
-2 i \bar{y}^{T} & B & 2 i \bar{x}^{T} \\
w & y & -\bar{u}
\end{array}\right)\left(\begin{array}{ccc}
a & 0 & 0 \\
-2 i a A \bar{b}^{T} & A & 0 \\
a\left(s-i b \bar{b}^{T}\right) & b & \bar{a}^{-1}
\end{array}\right) \\
& =\left(\begin{array}{ccc}
u-2 i x A \bar{b}^{T}+z\left(s-i b \bar{b}^{T}\right) & a^{-1} x A+a^{-1} z b & (a \bar{a})^{-1} z \\
\star & 2 i \bar{b}^{T} x A+A^{-1} B A+2 i \bar{b}^{T} z b+2 i A^{-1} \bar{x}^{T} b & \star \\
\star & \star & \star
\end{array}\right) \text {, }
\end{aligned}
$$

where we didn't explicit the action in the lower diagonal because this will not be used in the sequel. The following lemma follows from a simple computation using the formula above.

LEMMA 2.3. $A d\left(h^{-1}\right)\left(h_{1}\right) \subset h_{1}$ for $h \in H_{C R}$.

We have by the previous lemma the following

Lemma 2.4. The adjoint action of $H_{C R}$ on $g_{C R}$ passes to the quotient $v_{C R}$. 


\subsection{A bilinear form}

Using left invariant forms we construct the Maurer-Cartan form on $S U(n+1,1)$ with values in the Lie algebra $s u(n+1,1)$. That is a $g_{C R}$-valued form (we use Chern's coordinates).

$$
\Pi=\left(\begin{array}{ccc}
-\frac{1}{2} \phi-\frac{1}{n+2} \operatorname{Tr} \eta & \pi & 2 \omega \\
\star & \eta-\frac{1}{n+2} \operatorname{Tr} \eta I & 2 i \bar{\pi}^{T} \\
\star & \star & \frac{1}{2} \phi-\frac{1}{n+2} \operatorname{Tr} \eta
\end{array}\right)
$$

where $\pi=\left(\omega^{\beta}\right), \eta=\left(\eta_{\alpha}^{\beta}\right)$ and $\phi, \omega$ are real forms (the terms under the diagonal will not be used so we don't need to name them) and satisfying the following transformation law

$$
R_{h}^{*} \Pi=A d\left(h^{-1}\right) \Pi
$$

for $h \in P U(n+1,1)$. The Maurer Cartan equation is

$$
d \Pi+\Pi \wedge \Pi=0 .
$$

Observe that one of these equations is $2 d \omega=2 i \pi \wedge \bar{\pi}^{T}-2 \phi \wedge \omega$ which will appear as equation 4 in the general sub-conformal case. Chern's convention is made in order that the structure equation 7 be valid.

DEFinition 2.5. On $S U(n+1,1)$ define the form

$$
b=i \frac{2}{n+2} \operatorname{Tr} \eta \cdot \omega+\pi \cdot \bar{\pi}^{T}
$$

Proposition 2.1. $R_{h}^{*} b=|a|^{-2} b$ for $h \in H_{C R}$.

Proof. We use the adjoint action on $\Pi$ to compute the action on $b$.

That bilinear form is well defined, up to a conformal factor, in a quotient of the group $P U(n+1,1)$ diffeomorphic to the trivial circle bundle over the sphere $S^{2 n+1}$.

Proposition 2.2. $\quad b$ defines a conformal Lorentz structure on

$$
P U(n+1,1) / H_{1}=P U(n+1,1) / C S U(n) \ltimes N \simeq S^{2 n+1} \times S^{1}
$$

Proof. We have to show that $i\left(X^{*}\right) b=0$ for $X \in \operatorname{csu}(n) \ltimes N$, where $X^{*}(g)=\frac{d g e^{t X}}{d t}(0)$. That is equivalent to showing that $b$ vanishes along vectors tangent to the orbits of $C S U(n) \ltimes N$. The vector fields $X^{*}$ are left invariant and dual to the left invariant forms. In particular they are in the kernel of the forms Tr $\eta, \pi$ and $\omega$. 
The bilinear form $b$ is defined on $g_{C R} \otimes g_{C R}$ but, in fact, it uses only part of the left invariant forms in the group. It is most naturally defined on $v_{C R} \otimes v_{C R}$. That is the key observation which allows a more general construction.

\subsection{Real representation}

In this section we relate complex coordinates used by Chern to the real coordinates used in sub-conformal geometry. It assures that we are dealing with the same forms when the sub-conformal geometry gives rise to an integrable CR structure. A real representation of $H_{C R}$ is given by the following matrices

$$
\left(\begin{array}{ccc}
1 & 0 & 0 \\
2 h_{i r} a_{r}^{k} v^{k} & t a_{i}^{j} & 0 \\
s & t v^{j} & t^{2}
\end{array}\right)
$$

Here $a_{i}^{j} \in U(n)$ and $\left(h_{i r}\right)=J$ where $i$ is the row index and $r$ is the column index of the matrix. This group is isomorphic to $C U(n) \ltimes N$, the group of similarities of the Heisenberg group and an explicit isomorphism is given writing $a=t^{-1} e^{i \theta}$ and

$$
\begin{gathered}
\mathbf{a}=\left(\begin{array}{cc}
\Re e^{-i \theta} A & \Im e^{-i \theta} A \\
-\Im e^{-i \theta} A & \Re e^{-i \theta} A
\end{array}\right) \\
v=\left(\Re e^{-i \theta} b, \Im e^{-i \theta} b\right)
\end{gathered}
$$

by (observe that the kernel of the map is precisely the center of $P U(n+1,1)$ )

$$
\mu:\left(\begin{array}{ccc}
a & 0 & 0 \\
-2 i a A \bar{b}^{T} & A & 0 \\
a\left(s-i b \bar{b}^{T}\right) & b & \bar{a}^{-1}
\end{array}\right) \rightarrow\left(\begin{array}{ccc}
1 & 0 & 0 \\
4 J \mathbf{a} v^{T} & t \mathbf{a} & 0 \\
-4 s & 2 t v & t^{2}
\end{array}\right) .
$$

This map is obtained by comparing the right multiplication with the adjoint action on the algebra.

Proposition 2.3. The map

given by

$$
\frac{g^{0}}{u(n)} \oplus g^{-1} \oplus g^{-2} \rightarrow \mathbf{R} \oplus \mathbf{R}^{2 n} \oplus \mathbf{R}
$$

$$
(\phi, \pi, \omega) \rightarrow(\phi, \Re \pi, \Im \pi, \omega)
$$

(where $\phi=-\frac{\Re u}{2}$ and $\omega=\frac{z}{2}$ in the coordinates of the Lie algebra) is $H_{C R^{-}}$ equivariant with respect to the adjoint action on $\frac{g^{0}}{u(n)} \oplus g^{-1} \oplus g^{-2}$ and right
multiplication on $\mathbf{R} \oplus \mathbf{R}^{2 n} \oplus \mathbf{R}$.

Proof. By the adjoint action on the left hand side we obtain

$$
\begin{gathered}
\phi^{\prime}=\phi+2 i \pi A \bar{b}^{T}-2 i \bar{\pi} \bar{A} b^{T}-4 s \omega \\
\pi^{\prime}=a^{-1} \pi A+2 a^{-1} \omega b \\
\omega^{\prime}=(a \bar{a})^{-1} \omega
\end{gathered}
$$


A LORENTZ FORM ASSOCIATED TO CONTACT SUB-CONFORMAL AND CR MANIFOLDS 413

We write

$$
\begin{aligned}
\pi a^{-1} A+2 \omega a^{-1} b= & \left(\Re(\pi) \Re\left(a^{-1} A\right)-\Im(\pi) \Im\left(a^{-1} A\right)+2 \omega \Re\left(a^{-1} b\right)\right) \\
& +i\left(\Re(\pi) \Im\left(a^{-1} A\right)+\Im(\pi) \Re\left(a^{-1} A\right)+2 \omega \Im\left(a^{-1} b\right)\right)
\end{aligned}
$$

Also,

$$
\begin{aligned}
\phi+ & 2 i \pi A \bar{b}^{T}-2 i \bar{\pi} \bar{A} b^{T}-4 s \omega \\
& =\phi+(\Re \pi, \Im \pi)\left(\begin{array}{cc}
-4 \Im e^{-i \theta} A & 4 \Re e^{-i \theta} A \\
-\Re e^{-i \theta} A & -4 \Im e^{-i \theta} A
\end{array}\right)\left(\begin{array}{c}
\Re e^{-i \theta} b^{T} \\
\Im e^{-i \theta} b^{T}
\end{array}\right)-4 s \omega
\end{aligned}
$$

The Lie algebra of $H_{C R}$ in the real representation is given by matrices of the form

$$
\left(\begin{array}{ccc}
0 & 0 & 0 \\
* & t \delta_{i}^{j}+\omega_{i}^{j} & 0 \\
* & * & 2 t
\end{array}\right)
$$

where $\left(\omega_{i}^{j}\right) \in o(2 n)$.

In the next sections we need the equivalent to iTr $\eta$ using the real representation:

LEMMA 2.5. $i T r \eta=-\frac{1}{2} h^{i j} \omega_{j}^{i}$ where $h_{i j} h^{j k}=\delta_{i}^{k}$

Proof. We compute the differential of the homomorphism $\mu$ :

$$
\left(\begin{array}{ccc}
-\frac{1}{2} \phi-\frac{1}{n+2} T r \eta & \pi & 2 \omega \\
\star & \eta-\frac{1}{n+2} \operatorname{Tr} \eta I & 2 i \bar{\pi}^{T} \\
\star & \star & \frac{1}{2} \phi-\frac{1}{n+2} T r \eta
\end{array}\right) \rightarrow\left(\begin{array}{ccc}
0 & 0 & 0 \\
* & \frac{\phi}{2} I+\omega_{j}^{i} & 0 \\
* & * & \phi
\end{array}\right)
$$

where

$$
\omega_{j}^{i}=\left(\begin{array}{cc}
\Re \eta & \Im \eta \\
-\Im \eta & \Re \eta
\end{array}\right)
$$

therefore

$$
\begin{aligned}
h^{i j} \omega_{j}^{i} & =\operatorname{Tr}\left(-J\left(\begin{array}{cc}
\Re \eta & \Im \eta \\
-\Im \eta & \Re \eta
\end{array}\right)\right) \\
& =\operatorname{Tr}\left(\begin{array}{cc}
\Im \eta & 0 \\
0 & \Im \eta
\end{array}\right)=-2 i \operatorname{Tr} \eta
\end{aligned}
$$


Putting together the various transformations we obtain the following real representation corresponding to the adjoint action $\rho$ of $H_{C R}$ in $v_{C R}$.

Lemma 2.6. Let $v_{C R}=\{(\omega, \Re \pi, \Im \pi, \sigma)\}$. The representation $\rho$ of $H_{C R}$ on $v_{C R}$ is given by

$$
\begin{aligned}
& \rho\left(\left(\begin{array}{ccc}
1 & 0 & 0 \\
2 J \mathbf{a} v^{T} & t \mathbf{a} & 0 \\
s & t v & t^{2}
\end{array}\right)\right)(\omega, \Re \pi, \Im \pi, \sigma) \\
& \quad=\left(t^{2} \omega,(\Re \pi, \Im \pi) t \mathbf{a}+2 t v \omega, \sigma-2(\Re \pi, \Im \pi) \mathbf{a} v^{T}-v v^{T} \omega\right)
\end{aligned}
$$

\subsection{Principal fiber bundles, connections and invariant forms}

In this section we give an overview of the relevant bundles to be defined in detail in the next section. We will consider principal bundles $\pi: P \rightarrow M$ with a right action $P \times H \rightarrow P$. We usually denote the right action as $R_{h} p=p h$. Let $\omega$ be a form defined on $P$ with values in $V$, a vector space. Let $\rho: H \rightarrow G L(V)$ be a representation of $H$.

Definition 2.6. $\omega$ is a $\rho$-invariant form with values in $V$ if $R_{h}^{*} \omega=\rho\left(h^{-1}\right) \omega$ for $h \in H$.

Special cases of that definition include connection forms and Cartan connection forms defined on principal bundles. In the first case $V=h$, the Lie algebra of $H$ and in the second case $V$ is the Lie algebra of a group containing $H$. In both cases $\rho(h) v=A d(h) v$.

Given a sub-conformal structure on a manifold $M$ we construct a bundle $Y$ which factors through a $\mathbf{R}^{+}$-bundle denoted by $E$ (see next section). The bundle $Y$ is not a principal bundle but a reduction of $Y$ which we denote by $Y_{C R}$ will be principal with some extra hypothesis. In particular we will work with a bundle $Y_{C R} \rightarrow M$ which has $H_{C R}=C U(n) \ltimes N$ as structure group. Here $N$ is the Heisenberg group of dimension $2 n+1$ and $C U(n)$ is the conformal unitary group, that is, $\mathbf{R}^{+} \times U(n)$. Observe that $U(1)=C U(n) \ltimes N / C S U(n) \ltimes N$ so we obtain the circle bundle $\mathbf{T}=Y_{C R} / C S U(n) \ltimes N$ over $M$. The relevant bundles can be viewed in the following diagram.

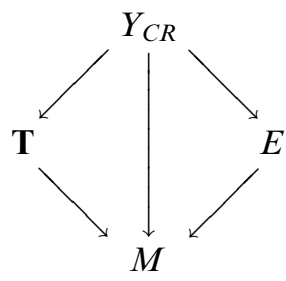

In the case that the sub-conformal structure gives rise to an integrable $\mathrm{CR}$ structure, the principal bundle $Y_{C R}$ is equipped with a Cartan connection. 
Although we are not going to use the following theorem, we state it for completeness and for comparison purposes. We won't state either the conditions defining uniquely that connection (referring to $[\mathrm{CM}]$ )

THEOREM 2.1 (Cartan-Tanaka-Chern). Over an integrable CR manifold $M$ there exists a bundle $Y_{C R}$ with a canonical $g_{C R}$-valued Cartan connection. That is a $g_{C R^{-v}}$ alued form $\Pi$ satisfying the following transformation law

$$
R_{h}^{*} \Pi=A d\left(h^{-1}\right) \Pi
$$

for $h \in H_{C R}$.

From the theorem above and Lemma 2.4 we easily get the following

COROLlary 2.1. Over an integrable CR manifold $M$ there exists a bundle $Y_{C R}$ with a canonical $v_{C R}$-valued invariant form.

The importance of that corollary is that the Lorentz form defined over $\mathbf{T}$ is constructed only using the $v_{C R}$-valued invariant form. Concretely one uses only part of the canonical connection to define the bilinear form. That means that even without a Cartan connection, one might be able to find an appropriate invariant form which would be enough to construct invariant bilinear forms. Moreover its construction is simplified, even in the integrable case, because we don't need to construct the whole Cartan connection.

In the next sections our goal will be to introduce the bundle $Y_{C R}$ and obtain an invariant bilinear form defined on $Y_{C R}$ for a general partially-integrable $\mathrm{CR}$ manifold. The main goal is theorem 4.1.

\section{Sub-conformal geometry}

This section is based on $[\mathrm{FV}]$ where a parallelism is obtained for subconformal geometry. Here we only need part of the forms constructed so we give a self-contained and simpler version of the forms we need.

Let $(M, D, \tilde{g})$ be a contact sub-conformal structure. Let $\tilde{E}$ to be the oriented line bundle of all sub-Riemannian metrics in the conformal class $\tilde{g}$. Given a sub-Riemannian metric, there exists a unique, up to sign, contact form $\theta$ such that

$$
d \theta=h_{i j} \theta^{i} \wedge \theta^{j}+h_{i} \theta^{i} \wedge \theta
$$

where $\theta^{i}$ is a dual basis of an orthonormal basis of $D, h_{i j}=-h_{j i}$. and $\operatorname{det}\left(h_{i j}\right)=1$ (observe that the determinant of a non-degenerate skew-symmetric matrix is positive so this condition does not depend on the choice of orientation on $D$ ). The matrix $\left(h_{i j}\right)$ is the matrix of the operator $H$ introduced in section 2 and we will denote it by the same letter $H$. We suppose from now on that $T M / D$ is oriented. We can therefore define the oriented line bundle $E$ of contact forms over $M . \quad \tilde{E}$ is isomorphic to $E$. 
We obtain in this way a set of basis of 1-forms adapted to the subRiemannian structure which can be described by all forms

$$
\begin{gathered}
\theta^{\prime}=\theta \\
\theta^{\prime i}=a_{j}^{i} \theta^{j}+v^{i} \theta
\end{gathered}
$$

where $a_{j}^{i}$ are orthonormal matrices and $v^{i}$ are arbitrary. Also, the change of matrix $\left(h_{i j}\right)$ is given by

$$
h_{i j}^{\prime}=a_{k}^{i} h_{k l} a_{l}^{j} .
$$

A natural coordinate on the line bundle is given by positive real numbers $\lambda$ so that $g=\frac{1}{\lambda} g_{0}$ where $g_{0}$ is a reference metric. The forms corresponding to the metric $g=\frac{1}{\lambda} g_{0}$ are given by

$$
\begin{gathered}
\theta^{\prime}=\lambda \theta \\
\theta^{i}=\sqrt{\lambda}\left(a_{j}^{i} \theta^{j}+v^{i} \theta\right)
\end{gathered}
$$

In order to work with the whole class of conformal metrics we consider the tautological forms defined by the forms above over the line bundle $E$. We write again

$$
\begin{gathered}
\omega^{\prime}=\omega \\
\omega^{i}=a_{j}^{i} \omega^{j}+v^{i} \omega
\end{gathered}
$$

where we understand that the forms are defined over $E$. Those forms vanish on vertical vectors, that is, vectors in the kernel of the map $T E \rightarrow T M$. In order to define non-horizontal 1 -forms we differentiate equation 2 to obtain

$$
d \omega=\omega \wedge \phi+h_{i j} \omega^{i} \wedge \omega^{j}
$$

where $\phi=-\frac{d \lambda}{\lambda}-h_{i} \theta^{i}$. Observe that $\frac{d \lambda}{\lambda}$ is a form intrinsically defined on $E$ up to horizontal forms (the minus sign is just a matter of conventions and makes the equations compatible with the $\mathrm{CR}$ case treated in $[\mathrm{CM}]$ ). In fact, choosing a different reference metric $g_{1}$ with $g_{0}=g_{1} / \mu$ where $\mu$ is a function over $M$, we can write $g=\frac{1}{\lambda} g_{0}=\frac{1}{\lambda \mu} g_{1}$ and obtain

$$
\frac{d(\lambda \mu)}{\lambda \mu}=\frac{d \lambda \mu+\lambda d \mu}{\lambda \mu}=\frac{d \lambda}{\lambda}+\frac{d \mu}{\mu},
$$

where $\frac{d \mu}{\mu}$ is a horizontal form. 
Definition 3.1. We denote by $Y$ the coframe bundle $Y \rightarrow E$ given by the set of 1 -forms $\phi, \omega^{j}, \omega$. Two coframes are related by

$$
\left(\phi^{\prime}, \omega^{i}, \omega^{\prime}\right)=\left(\phi, \omega^{j}, \omega\right)\left(\begin{array}{ccc}
1 & 0 & 0 \\
-2 v^{k} a_{r}^{k} h_{r j} & a_{j}^{i} & 0 \\
s & v^{i} & 1
\end{array}\right)
$$

where $a_{i}^{j}$ is an orthogonal matrix and $s, v^{i} \in \mathbf{R}$.

Considered as a bundle over $M$ the change of coframes in a fiber is given by the following matrix, where $t \in \mathbf{R}^{+}$,

$$
\left(\begin{array}{ccc}
1 & 0 & 0 \\
-2 v^{k} a_{r}^{k} h_{r j} & t a_{j}^{i} & 0 \\
s & t v^{i} & t^{2}
\end{array}\right) .
$$

The fact that the bundle $Y$ is not principal prevents us to obtain a simple description of the bundle.

In section 4 we use a reduction of the bundle $Y$ in a special case. We suppose that the canonical form $\Lambda$ of $H=\left(h_{i j}\right)$ as in lemma 2.1 is

$$
\Lambda=J=\left(\begin{array}{cc}
0 & I d \\
-I d & 0
\end{array}\right)
$$

That case gives rise to partially-integrable CR structures.

Definition 3.2. $Y_{C R}$ is the sub-bundle of $Y$ defined by the coframes such that $\left(h_{i j}\right)=J$, where $\Lambda$ is the normal form of lemma 2.1 .

$Y_{C R}$ is a principal bundle over $M$ with structure group $H_{C R}=C U(n) \ltimes N$, the group of similarities of the Heisenberg group.

\subsection{A distinguished form on subconformal structures}

In this section we construct the form we need, in the more general situation without hypothesis on $H$. We leave the study of its transformation to section 3.3. Given a sub-conformal manifold $M$ we construct the bundle $Y$ as in the previous section. We use the same notation $\omega^{i}, \omega$ and $\phi$ for the tautologous forms in $Y$ corresponding to the coframes defining the bundle.

We will use the following

LeMma 3.1. Let $\Omega^{\alpha}$ bet a set of 2-forms and $\omega^{\alpha}$ be a set of 1 -forms with the same number of elements satisfying $\Omega^{\alpha}=h_{\beta \gamma}^{\alpha} \omega^{\beta} \wedge \omega^{\gamma}$ with $h_{\beta \gamma}^{\alpha}=-h_{\gamma \beta}^{\alpha}$ then we can write $\Omega^{\alpha}=\omega^{\beta} \wedge \omega_{\beta}^{\alpha}$ with $\omega_{\beta}^{\alpha}=-\omega_{\alpha}^{\beta}$. If the set $\omega^{\alpha}$ is linearly independent, the forms $\omega_{\beta}^{\alpha}$ are unique. 
The lemma follows from a skew-symmetrization:

$$
\omega_{\beta}^{\alpha}=\left(h_{\beta \gamma}^{\alpha}-h_{\alpha \gamma}^{\beta}-h_{\alpha \beta}^{\gamma}\right) \omega^{\gamma} .
$$

Observe that in the case $\omega^{\alpha}$ is a linearly independent set the uniqueness follws from Cartan's trick as in the proof of the uniqueness of a Riemannian connection.

\subsubsection{The form $\sigma$}

Differentiating equation 3 we obtain

(6) $d \omega^{i}=\frac{d \lambda}{2 \lambda} \wedge \omega^{i}+a_{k}^{j} d a_{k}^{i} \wedge \omega^{j}+\frac{1}{\sqrt{\lambda}}\left(d v^{i}-a_{j}^{k} v^{k} d a_{j}^{i}\right) \wedge \omega+\sqrt{\lambda}\left(a_{j}^{i} d \theta^{j}+v^{j} d \theta\right)$,

Recall that, as $\left\{\theta^{j}, \theta\right\}$ is a basis of the cotangent space, one can write $\left\{d \theta^{j}, d \theta\right\}$ using those forms. Therefore one can write the last term using the tautological forms, modulo $\omega$, as

$$
\sqrt{\lambda}\left(a_{j}^{i} d \theta^{j}+v^{j} d \theta\right) \equiv h_{k j}^{i} \omega^{k} \wedge \omega^{j}
$$

with $h_{k j}^{i}=-h_{j k}^{i}$. Therefore, modulo $\omega$, we can write formula 6 as

$$
d \omega^{i} \equiv-\frac{1}{2} \phi \wedge \omega^{i}+a_{k}^{j} d a_{k}^{i} \wedge \omega^{j}+h_{k j}^{i} \omega^{k} \wedge \omega^{j} .
$$

As $a_{k}^{j} d a_{k}^{i}$ is skew-symmetric and using Lemma 3.1 we can further write

$$
d \omega^{i}=-\frac{1}{2} \phi \wedge \omega^{i}-\omega_{j}^{i} \wedge \omega^{j}-\phi^{i} \wedge \omega
$$

with $\omega_{j}^{i}=-\omega_{i}^{j}$.

Let $\omega_{j}^{i}, \bar{\omega}_{j}^{i}$ and $\phi^{i}, \bar{\phi}^{i}$ be forms satisfying the equation 7 . Taking the difference between the equations we obtain

$$
\left(\omega_{j}^{i}-\bar{\omega}_{j}^{i}\right) \wedge \omega^{j}+\left(\phi^{i}-\bar{\phi}^{i}\right) \wedge \omega=0
$$

and using Cartan's lemma we obtain

$$
\begin{gathered}
\omega_{j}^{i}-\bar{\omega}_{j}^{i}=c_{j}^{i} \omega \\
\phi^{i}-\bar{\phi}^{i}=c_{j}^{i} \omega^{j}+c^{i} \omega
\end{gathered}
$$

with

$$
c_{j}^{i}=-c_{i}^{j}
$$

Our objective is to define intrinsically and uniquely the form

$$
\sigma=-\frac{1}{n+2} h^{i j} \omega_{j}^{i}
$$

(where $h^{i j} h_{j k}=\delta_{k}^{i}$ ) by some normalization. Calling $\Omega=\left(\omega_{i}^{j}\right)$ and $H^{-1}=\left(h^{i j}\right)$ we have $\sigma=-\frac{1}{n+2} \operatorname{Tr}\left(H^{-1} \Omega\right)$. 
A LORENTZ FORM ASSOCIATED TO CONTACT SUB-CONFORMAL AND CR MANIFOLDS 419

\subsubsection{Fixing the form $\sigma$}

Differentiating equation 4 we obtain

$$
\left(d h_{i j}-h_{k j} \omega_{i}^{k}+h_{k i} \omega_{j}^{k}\right) \omega^{i} \wedge \omega^{j}-\left(d \phi-2 h_{i j} \phi^{i} \wedge \omega^{j}\right) \wedge \omega=0
$$

We need now the following simple lemma

Lemma 3.2. Let $A$ be a 2-form and $B_{i j}$ be 1-forms with $B_{i j}=-B_{i j}$ and $A \wedge \omega+B_{i j} \omega^{i} \wedge \omega^{j}=0$ then $A=-b_{i j} \omega^{i} \wedge \omega^{j}+\eta \wedge \omega$ and $B_{i j}=b_{i j k} \omega^{k}+b_{i j} \omega$ where $b_{i j}=-b_{j i}, b_{i j k}+b_{k i j}+b_{j k i}=0$ and $b_{i j k}=-b_{j i k}$.

Applying the lemma to equation 9 we obtain

$$
\begin{gathered}
d \phi-2 h_{i j} \phi^{i} \wedge \omega^{j}+\eta \wedge \omega=b_{i j} \omega^{i} \wedge \omega^{j} \\
d h_{i j}-h_{k j} \omega_{i}^{k}+h_{k i} \omega_{j}^{k}=b_{i j k} \omega^{k}+b_{i j} \omega
\end{gathered}
$$

If we use 8 in 11 above we obtain

$$
\left(h_{j k} c_{i}^{k}+h_{k i} c_{j}^{k}\right) \omega=\left(b_{i j k}-\bar{b}_{i j k}\right) \omega^{k}+\left(b_{i j}-\bar{b}_{i j}\right) \omega
$$

Then

$$
\begin{gathered}
b_{i j k}=\bar{b}_{i j k} \\
h_{j k} c_{i}^{k}-h_{i k} c_{j}^{k}=b_{i j}-\bar{b}_{i j}
\end{gathered}
$$

If we differentiate the equation 7 and using 10, 7 and 4, we obtain

$$
\begin{aligned}
& \left(d \phi^{i}-\frac{1}{2} \phi \wedge \phi^{i}-\phi^{j} \wedge \omega_{j}^{i}+\frac{1}{2} \eta \wedge \omega^{i}\right) \wedge \omega \\
& \quad+\left(d \omega_{j}^{i}+\omega_{l}^{i} \wedge \omega_{j}^{l}+h_{l j} \omega^{i} \wedge \phi^{l}-\frac{1}{2} b_{l j} \omega^{l} \wedge \omega^{i}-h_{l j} \phi^{i} \wedge \omega^{l}\right) \wedge \omega^{j}=0
\end{aligned}
$$

It follows that

$$
\Phi_{j}^{i} \wedge \omega^{j} \equiv 0 \quad \bmod \omega
$$

where

$$
\Phi_{j}^{i}=d \omega_{j}^{i}+\omega_{l}^{i} \wedge \omega_{j}^{l}+W_{j k l}^{i} \omega^{k} \wedge \phi^{l}+B_{j k l}^{i} \omega^{k} \wedge \omega^{l}
$$

with

$$
W_{j k r}^{i}=\delta_{r}^{i} h_{k j}+\delta_{k}^{i} h_{r j}-\delta_{r}^{j} h_{k i}-\delta_{k}^{j} h_{r i}+\delta_{k}^{r} h_{i j}
$$

and

$$
B_{j k l}^{i}=\frac{1}{4}\left(b_{l j} \delta_{k}^{i}-b_{k j} \delta_{l}^{i}-b_{l i} \delta_{k}^{j}+b_{k i} \delta_{l}^{j}\right) .
$$

Observe that $W_{j k r}^{i}=-W_{i k r}^{j}=W_{j r k}^{i}, B_{j k r}^{i}=-B_{i k r}^{j}=-B_{j r k}^{i}$ and

$$
\Phi_{j}^{i}+\Phi_{i}^{j}=0 .
$$


From 13 and 14 follows easily the following lemma

LEMMA 3.3.

$$
\Phi_{j}^{i} \equiv S_{j k l}^{i} \omega^{k} \wedge \omega^{l} \quad \bmod \omega
$$

where $S_{j k l}^{i}=-S_{i k l}^{j}=-S_{j l k}^{i}$ and $S_{j k l}^{i}+S_{l j k}^{i}+S_{k l j}^{i}=0$.

The form $\Phi_{j}^{i}$ is not well determined. If we consider two sets of forms $\omega_{j}^{i}, \phi^{i}$ and $\bar{\omega}_{j}^{i}, \bar{\phi}^{i}$ as in 8, with the corresponding $\Phi_{j}^{i}$ and $\bar{\Phi}_{j}^{i}$ then

$$
\Phi_{j}^{i}-\bar{\Phi}_{j}^{i} \equiv W_{j k l}^{i} \omega^{k} \wedge\left(\phi^{l}-\bar{\phi}^{l}\right)+\left(c_{j}^{i} h_{k l}+B_{j k l}^{i}-\bar{B}_{j k l}^{i}\right) \omega^{k} \wedge \omega^{l} \bmod \omega .
$$

We are not going to determine the form $\Phi_{j}^{i}$ but only a certain trace of it. In order to do so let

$$
S_{k l}=\sum h^{i j} S_{j k l}^{i}
$$

and

$$
S=\sum S_{k l} h^{l k}
$$

In $[\mathrm{FV}]$ we used a different definition for $S$ and we show in the following section that the two definitions give the same normalization in the case of an integrable $\mathrm{CR}$ structure.

Proposition 3.1. The forms $\omega_{j}^{i}$ can be chosen so that $S=0$ and then the form

$$
\sigma=-\frac{1}{n+2} h^{i j} \omega_{j}^{i}
$$

is independent on the remaining choices.

Proof. The form $h^{i j} \omega_{j}^{i}$ is determined up to $c_{l}^{k} h^{l k}$. Taking into account that $h^{i j} W_{j k r}^{i}=-(2 n+4) \delta_{k}^{r}, h^{i j} B_{j k l}^{i}=0$ and using the expression of $S_{j k l}^{i}-\bar{S}_{j k l}^{i}$ obtained by skew-symmetrizing the formula for $\Phi_{j}^{i}-\bar{\Phi}_{j}^{i}$, that is,

$$
S_{j k l}^{i}-\bar{S}_{j k l}^{i}=\frac{1}{2}\left(W_{j k r}^{i} c_{l}^{r}-W_{j l r}^{i} c_{k}^{r}\right)+c_{j}^{i} h_{k l}+B_{j k l}^{i}-\bar{B}_{j k l}^{i}
$$

we get

$$
S-\bar{S}=(4 n+4) c_{l}^{k} h^{l k}
$$

and this determines uniquely $c_{l}^{k} h^{l k}$ such that $S=0$.

Using the 1-form $\sigma$ we may now define the bilinear form which will give rise to a conformal Lorentz structure in the case of a partially integrable CR structure. 
DeFINITION 3.3. Define on $Y$ the bilinear form

$$
b=\omega^{i} \omega^{i}+\sigma \omega
$$

\subsection{Relation to integrable $C R$ structures}

In $[\mathrm{FV}]$ we used a different definition for $S$ (cf. Lemma 4.4 pg. 462). In this section we show that the two definitions give the same normalization in the case of an integrable CR structure. This implies that the form $\sigma$ defined in section 3 coincides with the one defined in [BDS] in that case.

It is shown in [FV] (Proposition 5.1 pg. 472) that the sub-conformal parallelism coincides with the parallelism obtained from the Cartan connection defined in $[\mathrm{CM}]$ in the $\mathrm{CR}$ integrable case.

Following formula 41 and Lemma 5.4 in [FV] we may suppose for each fixed pair $k, l, S_{j k l}^{i} \in u(n)$, that is, $S_{\beta k l}^{\alpha}=S_{\beta+n k l}^{\alpha+n}$ and $S_{\beta k l}^{\alpha+n}=-S_{\beta+n k l}^{\alpha}$, for $0 \leq \alpha, \beta \leq n$.

Lemma 3.4. If $\left(h_{i j}\right)=J$ then

$$
h^{i j} S_{j k l}^{i} h^{k l}=2 S_{j i j}^{i}
$$

Proof. We write $h^{i j} S_{j k l}^{i} h^{k l}=-h^{i j}\left(S_{k l j}^{i}+S_{l j k}^{i}\right) h^{k l}$. After a permutation of the indices we obtain

$$
=-h^{i j} S_{k l j}^{i} h^{k l}-h^{k l} S_{i l j}^{k} h^{j i}=2 h^{i j} S_{k j l}^{i} h^{k l} .
$$

Using $S_{j k l}^{i} \in u(n)$ we obtain

$$
\begin{aligned}
& 2\left(h^{\alpha \alpha+n} S_{\beta \alpha+n \beta+n}^{\alpha} h^{\beta \beta+n}+h^{\alpha \alpha+n} S_{\beta+n \alpha+n \beta}^{\alpha} h^{\beta+n \beta}\right. \\
&\left.+h^{\alpha+n \alpha} S_{\beta \alpha \beta+n}^{\alpha+n} h^{\beta \beta+n}+h^{\alpha+n \alpha} S_{\beta+n \alpha \beta}^{\alpha+n} h^{\beta+n \beta}\right) \\
&= 2\left(S_{\beta \alpha+n \beta+n}^{\alpha}-S_{\beta+n \alpha+n \beta}^{\alpha}-S_{\beta \alpha \beta+n}^{\alpha+n}+S_{\beta+n \alpha \beta}^{\alpha+n}\right) \\
&= 2\left(S_{\beta+n \alpha+n \beta+n}^{\alpha+n}+S_{\beta \alpha+n \beta}^{\alpha+n}+S_{\beta+n \alpha \beta+n}^{\alpha}+S_{\beta \alpha \beta}^{\alpha}\right) \\
&= 2 S_{j i j}^{i}
\end{aligned}
$$

Observe that this shows that the normalization $h^{i j} S_{j k l}^{i} h^{k l}=0$ is equivalent to the normalization $S_{j i j}^{i}=0$ and therefore that our form $\sigma$ coincides with the one defined by $[\mathrm{BDS}]$ using the Cartan connection.

\subsection{Change of Coframe}

Our goal is to obtain the transformation of $\sigma=-\frac{1}{n+2} h^{i j} \omega_{j}^{i}$ when we compute it in a new basis of forms

$$
\begin{aligned}
\omega^{\prime} & =t^{2} \omega \\
\omega^{i} & =t\left(a_{j}^{i} \omega^{j}+v^{i} \omega\right) \\
\phi^{\prime} & =\phi-2 v^{k} a_{r}^{k} h_{r j} \omega^{j}+s \omega
\end{aligned}
$$


on $Y$ as given by the right action of the group $H_{C R}$ represented by matrices of the form 5 .

We have the analogous equations

$$
\begin{gathered}
d \omega^{\prime}=\omega^{\prime} \wedge \phi^{\prime}+h_{i j}^{\prime} \omega^{\prime i} \wedge \omega^{\prime j} \\
d \omega^{\prime i}=-\frac{1}{2} \phi^{\prime} \wedge \omega^{\prime i}-\omega_{j}^{\prime i} \wedge \omega^{\prime j}-\phi^{\prime i} \wedge \omega^{\prime} \\
d h_{i j}^{\prime}-h_{k j}^{\prime} \omega_{i}^{\prime k}+h_{k i}^{\prime} \omega_{j}^{\prime k}=b_{i j k}^{\prime} \omega^{\prime k}+b_{i j}^{\prime} \omega^{\prime}
\end{gathered}
$$

Differentiating equation $\omega^{i}=t\left(a_{j}^{i} \omega^{j}+v^{i} \omega\right)$ we obtain

$$
\begin{aligned}
-\frac{1}{2} \phi^{\prime} & \wedge \omega^{i}-\omega_{j}^{i} \wedge \omega^{j}-\phi^{i} \wedge \omega^{\prime} \\
= & t\left(a_{j}^{i} d \omega^{j}+v^{i} d \omega\right) \\
& =t v^{i}\left(\omega \wedge \phi+h_{k l} \omega^{k} \wedge \omega^{l}\right)+t a_{j}^{i}\left(-\frac{1}{2} \phi \wedge \omega^{j}-\omega_{k}^{j} \wedge \omega^{k}-\phi^{j} \wedge \omega\right)
\end{aligned}
$$

Using the inverse transformations

$$
\begin{aligned}
\omega & =t^{-2} \omega^{\prime} \\
\omega^{k} & =a_{k}^{i}\left(t^{-1} \omega^{\prime i}-t^{-2} v^{i} \omega^{\prime}\right) \\
\phi & =\phi^{\prime}+2 v^{k} t^{-1} h_{k j}^{\prime} \omega^{\prime j}-s t^{-2} \omega^{\prime}
\end{aligned}
$$

in the equation above and reorganizing we obtain

$$
\begin{aligned}
0= & {\left[\omega_{j}^{\prime i}-a_{l}^{i} \omega_{k}^{l} a_{k}^{j}+\frac{1}{t} v^{k} W_{j k r}^{\prime i} \omega^{\prime r}\right] \wedge \omega^{\prime j} } \\
& +\left[\phi^{\prime i}-\frac{1}{t}\left(a_{j}^{i} \phi^{j}+\frac{1}{2} v^{i} \phi^{\prime}-v^{l} a_{j}^{i} \omega_{k}^{j} a_{k}^{l}\right)+\frac{1}{t^{2}}\left(v^{i} v^{k} h_{k l}^{\prime}-\frac{1}{2} \delta_{l}^{i} s\right) \omega^{\prime l}\right] \wedge \omega^{\prime}
\end{aligned}
$$

where $W_{j k r}^{\prime i}$ is the corresponding function to $W_{j k r}^{i}$ with $h_{i j}$ replaced by $h_{i j}^{\prime}$. It follows from Cartan's lemma that

$$
\omega_{j}^{\prime i}=a_{l}^{i} \omega_{k}^{l} a_{k}^{j}-\frac{1}{t} v^{k} W_{j k r}^{\prime i} \omega^{\prime r}+\frac{1}{t^{2}} d_{j}^{i} \omega^{\prime}
$$

and

$$
\phi^{\prime i}=\frac{1}{t}\left(a_{j}^{i} \phi^{j}+\frac{1}{2} v^{i} \phi^{\prime}-v^{l} a_{j}^{i} \omega_{k}^{j} a_{k}^{l}\right)-\frac{1}{t^{2}}\left(v^{i} v^{k} h_{k l}^{\prime}-\frac{1}{2} \delta_{l}^{i} s-d_{l}^{i}\right) \omega^{\prime l}+c^{i} \omega^{\prime}
$$

with $d_{j}^{i}=-d_{i}^{j}$. Substituting 18 in 17 and taking into account equatons 11 and 1 we obtain

$$
\begin{aligned}
b_{i j k}^{\prime} \omega^{\prime k}+b_{i j}^{\prime} \omega^{\prime}= & \frac{1}{t}\left(a_{r}^{i} b_{r s t} a_{s}^{j} a_{t}^{k}+v^{r}\left(h_{s j}^{\prime} W_{i r k}^{\prime s}-h_{s i}^{\prime} W_{j r k}^{\prime s}\right)\right) \omega^{\prime k} \\
& +\frac{1}{t^{2}}\left(a_{r}^{i} b_{r s} a_{s}^{j}-a_{r}^{i} b_{r s t} a_{s}^{j} a_{t}^{k} v^{k}+h_{k i}^{\prime} d_{j}^{k}-h_{k j}^{\prime} d_{i}^{k}\right) \omega^{\prime} .
\end{aligned}
$$


A LORENTZ FORM ASSOCIATED TO CONTACT SUB-CONFORMAL AND CR MANIFOLDS 423

It follows that

$$
b_{i j}^{\prime}=\frac{1}{t^{2}}\left(a_{r}^{i} b_{r s} a_{s}^{j}-a_{r}^{i} b_{r s t} a_{s}^{j} a_{t}^{k} v^{k}+h_{k i}^{\prime} d_{j}^{k}-h_{k j}^{\prime} d_{i}^{k}\right)
$$

and

$$
b_{i j k}^{\prime}=\frac{1}{t}\left(a_{r}^{i} b_{r s t} a_{s}^{j} a_{t}^{k}+v^{r}\left(h_{s j}^{\prime} W_{i r k}^{\prime s}-h_{s i}^{\prime} W_{j r k}^{\prime s}\right)\right)
$$

Let's now see how $S_{j k l}^{i}$ changes. Inserting equations 18 and 19 in

$$
\Phi_{j}^{\prime i}=d \omega_{j}^{\prime i}+\omega_{l}^{\prime i} \wedge \omega_{j}^{\prime l}+W_{j k l}^{\prime i} \omega^{\prime k} \wedge \phi^{\prime l}+B_{j k l}^{\prime i} \omega^{\prime k} \wedge \omega^{\prime l}
$$

where $B_{j k l}^{\prime i}$ are the corresponding functions $B_{j k l}^{i}$ with primes, we get after some manipulation

$$
\begin{gathered}
S_{j k l}^{\prime i}=a_{x}^{i} a_{y}^{j} a_{r}^{k} a_{s}^{l} S_{y r s}^{x}+\frac{1}{2 t} v^{r}\left[\frac{1}{2} \delta_{k}^{i}\left(b_{r j l}^{\prime}+b_{r l j}^{\prime}\right)+\frac{1}{2} \delta_{l}^{i}\left(b_{j r k}^{\prime}+b_{k r j}^{\prime}\right)+\frac{1}{2} \delta_{k}^{j}\left(b_{i r l}^{\prime}+b_{l r i}^{\prime}\right)\right. \\
\left.+\frac{1}{2} \delta_{l}^{j}\left(b_{r i k}^{\prime}+b_{r k i}^{\prime}\right)+\delta_{r}^{i} b_{k l j}^{\prime}+\delta_{r}^{j} b_{l k i}^{\prime}+\delta_{r}^{k} b_{i j l}^{\prime}+\delta_{r}^{l} b_{j i k}^{\prime}\right] \\
+\frac{1}{2 t^{2}} v^{x} v^{y}\left[W_{r x k}^{\prime i} W_{r y l}^{\prime j}-W_{r x l}^{\prime i} W_{r y k}^{\prime j}+W_{j k r}^{\prime i} W_{r y l}^{\prime x}-W_{j l r}^{\prime i} W_{r y k}^{\prime x}-W_{j k x}^{\prime i} h_{y l}^{\prime}\right. \\
+W_{j l x}^{\prime i} h_{y k}^{\prime}+\frac{1}{2}\left(W_{l x y}^{\prime r} h_{r j}^{\prime}-W_{j x y}^{\prime r} h_{r l}^{\prime}\right) \delta_{k}^{i}+\frac{1}{2}\left(W_{i x y}^{\prime r} h_{r l}^{\prime}-W_{l x y}^{\prime r} h_{r i}^{\prime}\right) \delta_{k}^{j} \\
\left.-\frac{1}{2}\left(W_{k x y}^{\prime r} h_{r j}^{\prime}-W_{j x y}^{\prime r} h_{r k}^{\prime}\right) \delta_{l}^{i}-\frac{1}{2}\left(W_{i x y}^{\prime r} h_{r k}^{\prime}-W_{k x y}^{\prime r} h_{r i}^{\prime}\right) \delta_{l}^{j}\right] \\
+\left[d_{j}^{i} h_{k l}^{\prime}+\frac{1}{2} W_{j k x}^{\prime i} d_{l}^{x}-\frac{1}{2} W_{j l x}^{\prime i} d_{k}^{x}+\frac{1}{4}\left(\delta_{k}^{i} \delta_{j}^{m}-\delta_{k}^{j} \delta_{i}^{m}\right)\left(h_{r l}^{\prime} d_{m}^{r}-h_{r m}^{\prime} d_{l}^{r}\right)\right. \\
\left.-\frac{1}{4}\left(\delta_{l}^{i} \delta_{j}^{m}-\delta_{l}^{j} \delta_{i}^{m}\right)\left(h_{r k}^{\prime} d_{m}^{r}-h_{r m}^{\prime} d_{k}^{r}\right)\right]
\end{gathered}
$$

We analyze now how the form $h^{i j} \omega_{j}^{i}$ changes with the new referential.

PROPOSITION 3.2.

$$
\sigma^{\prime}=\sigma-\frac{1}{n+2}\left(\frac{2}{t}(n+2) v^{r} \omega^{\prime r}+\frac{1}{t^{2}} h^{\prime i j} d_{j}^{i} \omega^{\prime}\right)
$$

where

$$
\begin{aligned}
\frac{1}{t^{2}} h^{\prime i j} d_{j}^{i}= & \frac{1}{4(n+1)}\left[t v^{r}\left(2 h^{\prime k j} h^{\prime k l} b_{r j l}^{\prime}+2 h^{\prime r j} h^{\prime k l} b_{k j l}^{\prime}+h^{\prime i j} h^{\prime r l} b_{i j l}^{\prime}\right)\right. \\
& \left.-v^{x} v^{y}\left(\left(4 n^{2}+14 n+8\right) \delta_{y}^{x}-h^{\prime k l} h^{\prime k l} h_{x r}^{\prime} h_{y r}^{\prime}\right)\right]
\end{aligned}
$$


Proof. It follows from equation 18 and $h^{\prime i j} W_{j k r}^{\prime i}=-(2 n+4) \delta_{k}^{r}$ that

$$
h^{\prime i j} \omega_{j}^{\prime i}=h^{i j} \omega_{j}^{i}+\frac{2}{t}(n+2) v^{r} \omega^{\prime r}+\frac{1}{t^{2}} h^{\prime i j} d_{j}^{i} \omega^{\prime} .
$$

The second equation follows from $h^{\prime i j} S_{j k l}^{i j} h^{\prime k l}=h^{i j} S_{j k l}^{i} h^{k l}=0$.

\section{The bilinear form on $Y_{C R}$}

At this point we need to introduce the reduction $Y_{C R}$ of the bundle $Y$ assuming that $H=J$ on $Y_{C R}$ throughout this section. The main goal is Theorem 4.1 which describes a conformal Lorentz structure on a circle bundle over a partially-integrable CR structure.

Proposition 4.1. On $Y_{C R}$ we have

$$
\sigma^{\prime}=\sigma-2 v^{r} a_{k}^{r} \omega^{k}-v^{r} v^{r} \omega
$$

Proof. We first obtain the following formula on $Y_{C R}$ :

$$
h^{\prime i j} d_{j}^{i}=-(n+2) v^{x} v^{x} .
$$

In fact, from equation 11 we obtain that

$$
\begin{aligned}
& H^{-1} d H+d H H^{-1}+H^{-1} \Omega H-H \Omega H^{-1} \\
& \quad=\left(H^{-1} B_{l}+B_{l} H^{-1}\right) \omega^{l}+\left(H^{-1} B+B H^{-1}\right) \omega,
\end{aligned}
$$

where $\Omega=\left(\omega_{j}^{i}\right), B_{l}=\left(b_{i j l}\right)$, and $B=\left(b_{i j}\right)$. It follows from the above formula that $H^{-1} B_{l}+B_{l} H^{-1}=0$, or $h^{\prime k j} b_{r j l}^{\prime}+h^{\prime r j} b_{k j l}^{\prime}=0$ and $h^{\prime i j} b_{i j l}^{\prime}=0$. Also $h^{\prime k l} h^{\prime k l} h_{x r}^{\prime} h_{y r}^{\prime}=-2 n \delta_{y}^{x}$.

From the formula above we obtain the transformation properties of $\sigma=$ $-\frac{1}{n+2} h^{i j} \omega_{j}^{i}$ and complete the proof.

Consider the $v_{C R}$-valued form given by $\left(\omega, \omega^{i}, \sigma\right), 1 \leq i \leq 2 n$. We consider the action of $C U(n) \ltimes N$ on $v_{C R}$ as defined in section 2. Comparing the previous transformation laws and Lemma 2.6 we proved

Proposition 4.2. On a partially-integrable $Y_{C R}$ the $v_{C R}$-valued form $\left(\omega, \omega^{i}, \sigma\right)$ is $\rho$ invariant.

We consider the bilinear form $b=\omega^{i} \omega^{i}+\sigma \omega$ on $Y_{C R}$. It follows easily from proposition 4.2 that the form $b$ is invariant up to scalar multiple:

Proposition 4.3. On $Y_{C R}$,

$$
b^{\prime}=t^{2} b
$$


A LORENTZ FORM ASSOCIATED TO CONTACT SUB-CONFORMAL AND CR MANIFOLDS 425

Recall that $\mathbf{T}=Y_{C R} / C S U(n) \ltimes N$ is a circle bundle. We are ready to prove that the bilinear form defined above descends to that circle bundle.

THEOREM 4.1. If $M$ is a partially-integrable $C R$ manifold, the bilinear form on $Y_{C R}$

$$
b=\omega^{i} \omega^{i}+\sigma \omega
$$

descends to a Lorentz conformal structure on $\mathbf{T}=Y_{C R} / C S U(n) \ltimes N$. only if

Proof. A tensor $\varpi$ on $Y_{C R}$ projects to $\mathbf{T}$ up to a conformal factor if and

1. $R_{g}^{*} \varpi=f \varpi$ for every $g \in C S U(n) \ltimes N$,

2. $i\left(X^{*}\right)(\varpi)=0$ for every $X \in h_{1}=\operatorname{Lie}(\operatorname{CSU}(n) \ltimes N)$

We have already shown that $R_{g}^{*} b=t^{2} b$. As $X^{*}$ is vertical and $\omega^{i}$ and $\omega$ are tautological we have $\omega^{i}\left(X^{*}\right)=\omega\left(X^{*}\right)=0$. Then $i\left(X^{*}\right)(b)=\sigma\left(X^{*}\right) \omega$. It remains to show that

$$
h^{i j} \omega_{j}^{i}\left(X^{*}\right)=0,
$$

for $X \in h_{1}$. From equation 6 and 8 we obtain

$$
\omega_{j}^{i}=-a_{k}^{j} d a_{k}^{i}+c_{j k}^{i} \omega^{k}+c_{j}^{i} \omega .
$$

If $X \in h_{1}$ then

$$
\omega_{j}^{i}\left(X^{*}\right)=-a_{k}^{j} d a_{k}^{i}\left(X^{*}\right) \in \operatorname{csu}(n),
$$

therefore $h^{i j} \omega_{j}^{i}\left(X^{*}\right)=0$.

\section{REFERENCES}

[BDS] D. Burns, K. Diederich AND S. Shnider, Distinguished curves in pseudoconvex boundaries, Duke Math. J. 44 (1977), 407-431.

[BS] D. Burns AND S. Shnider, Real hypersurfaces in complex manifolds, Proc. sympos. pure math. 30, Amer. Math. Soc., Providence, RI, 1977, 141-168.

[Ca] A. CAP, Parabolic geometries, CR-tractors, and the Fefferman construction, Differential Geom. Appl. 17 (2002), 123-138.

[C] E. CARTAN, Sur la géométrie pseudo-conforme des hypersurfaces de deux variables complexes, I. Ann. Math. Pura Appl. 11 (1932), 17-90 (or Oeuvres II, 2 1231-1304), II, Ann. Scuola Norm. Sup. Pisa 1 (1932), 333-354 (or Oeuvres III, 2 1217-1238).

[CM] S. S. Chern AND J. Moser, Real hypersurfaces in complex manifolds, Acta Math. 133 (1974), 219-271.

[D] S. Dragomir, Pseudohermitian geometry, Bull. Math. Soc. Sci. Math. Roumanie 43(93) (2000), 225-234.

[FV] E. Falbel and J. M. Veloso, A parallelism for contact conformal sub-Riemannian geometry, Forum Mathematicum 10 (1998), 453-478.

[Fa] F. FARrIs, An intrinsic construction of Fefferman's CR metric, Pacific J. Math. 123 (1986), 33-45. 
[F] C. Fefferman, Monge-Ampère equations, the Bergman kernel, and the geometry of pseudoconvex domains, Ann. of Math. 103 (1976), 395-416, Correction 104 (1976), 393-394.

[K] S. Kobayashi, Transformation groups in differential geometry, Springer-Verlag, 1972.

[Ku] M. Kuranishi, CR geometry and Cartan geometry, Forum Math. 7 (1995), 147-206.

[L] J. M. LeE, The Fefferman metric and pseudo Hermitian invariants, Trans. Am. Math. Soc. 296 (1986), 411-429.

[M] T. MоRiмото, Geometric structures on filtered manifolds, Hokkaido Math. J. 22 (1993), 263-347.

[T1] N. TANAKA, Graded Lie algebras and geometrical structures, Proc. U.S.-Japan Seminar in Differential Geometry, Kyoto, Japan, 1965, 147-150.

[T2] N. TANAKA, On non-degenerate real hypersurfaces, graded Lie algebras and Cartan connections, Japan J. Math. 2 (1976), 131-190.

[T3] N. TanaKa, On the equivalence problem associated with simple graded Lie algebras, Hokkaido Math. J. 8 (1979), 23-84.

Elisha Falbel

Institut DE MathÉmatiques

ANAlyse COMPleXe et GÉOMÉTRIE

4 Place Jussieu, Paris

FRANCE

E-mail: falbel@math.jussieu.fr

José Miguel Veloso

FACUldade de Matemática-ICEN

Universidade Federal do ParÁ

66059 BelÉM, PA

BRAZIL

E-mail: veloso@ufpa.br 